J. Clin. Chem. Clin. Biochem.

Vol. 17, 1979, pp. 363-367

\title{
Eine radioenzymatische Mikromethode zur Bestimmung der UDP-Glucuronsäure
}

\author{
Von W. Lehnert, Th. O. F. Wagner, M. W. Fürst und W. Künzer \\ Univ.-Kinderklinik, (Direktor: Prof. Dr. W. Künzer) Freiburg i. Br.
}

(Eingegangen am 10. August 1978/.6. Februar 1979)

Zusammenfassung: Es wird eine neue empfindliche und spezifische Mikromethode zur Bestimmung der UDP-Glucuronsäure beschrieben. Hierbei werden Extrakte aus $2,5 \mathrm{mg}$ Gewebe mit Hilfe der UDP-Glucuronyltransferase aus gewaschenen Meerschweinchenmikrosomen mit $\left[{ }^{14} \mathrm{C}\right] p$-Nitrophenol inkubiert und der Umsatz an UDP-Glucuronsäure aus dem Verhältnis von entstandenem $\left[{ }^{14} \mathrm{C}\right] p$-Nitrophenylglucuronid und überschüssigem $\left[{ }^{14} \mathrm{C}\right] p$-Nitrophenol sowie der eingesetzten Menge an [ $\left.{ }^{14} \mathrm{C}\right] p$-Nitrophenol bestimmt. Verluste durch nicht vollständige Umsetzung und Nebenreaktionen werden durch einen Kalibrierwert, der zum normalen Inkubationsansatz zusätzlich eine bekannte Menge UDP-Glucuronsäure enthält, kompensiert. Für eine Bestimmung reichen Gewebsmengen, wie sie z. B. durch Leberblindpunktion bei Neugeborenen erhalten werden können $(5-10 \mathrm{mg})$. Der Variationskoeffizient der Bestimmungen beträgt $\pm 2.3 \%$. Der UDP-Glucuronsäure-Gehalt der Mäuseleber wurde zu $171 \mu \mathrm{mol} / \mathrm{kg}(\mathrm{n}=6)$ bestimmt.

\section{Radioenzymatic micromethod for the determination of UDP-ghucuronic acid}

Summary: A new sensitive and specific micromethod for determination of UDP-glucuronic acid is described. Extracts from $2.5 \mathrm{mg}$ of liver are incubated with twice washed guinea pig microsomes (as a source of UDP-glucuronyl transferase) and $\left[{ }^{14} \mathrm{C}\right] p$-nitrophenol. The content of UDP-glucuronic acid is calculated from the $\left[{ }^{14} \mathrm{C}\right] p$-nitrophenyl glucuronide $/\left[{ }^{14} \mathrm{C}\right] p$-nitrophenol radioactivity ratio and the known amount of introduced $\left[{ }^{14} \mathrm{C}\right] p$-nitrophenol. These values are corrected for losses of UDP-glucuronic acid by a calibration experiment, containing, in addition to the aforementioned constituents, a known amount of UDP-glucuronic acid. The mean concentration of UDP-glucuronic acid in mice liver was found to be $171 \mu \mathrm{mol} / \mathrm{kg}$ wet weight.

\section{Einfuihrung}

Für die Entstehung des physiologischen Neugeborenenikterus werden vornehmlich drei Gründe verantwortlich gemacht:

1. Störungen in der Aufnahme des Bilirubins durch die Leber,

2. die Ünreife der Ủ̉P-Glucuronyltransferase, die das Bilirubin mit Glucuronsäure koppelt und dadurch gallengängig macht, sowie

3. Exkretiónsstörungen der Leber.

Daneben gibt es Anhaltspunkte für eine verminderte Aktivität der UDP-Glucose-Dehy drogenase in der perinatalen Leber $(1,2)$. Da die UDP-Glucose aber Vorläufer der UDP-Glucuronsäure ist, sollte auch ein Mangel an ÜDP-Glucuronsäure in Erwägung gezogen werden. Diese Möglichkeit ist von Schröter \& Eggerling (3) sowie von
Felsher et al. (2) in diesem Zusammenhang bereits diskutiert worden.

Die vorliegende Arbeit soll zu diesem Thems insofern einen Beitrag leisten, als eine empfindliche und spezifische Mikrome thode zur Bestimmung der UDP-Glucuronsäure beschrieben wird, zu deren Durchführung schon 5-10 mg Gewebe ausreichen. Bisher sind UDP-Glucuronsäure-Bestimmungen nur vereinzelt beschrieben worden. So setzten Wong \& Sourkes (4) einen durch Kochen, Homogenisieren und Zentrifugieren gewonnenen Leberextrakt in Gegenwart von UDP-Glucuronyltransferase mit Harmol um und bestimmten das gebildete Glucuronid nach Dünnschichtchromatographie fluoreszenzspektroskopisch. Die benötigte Lebermenge betrug ca. $100 \mathrm{mg} /$ Bestimmungsansatz. Gegen das Kochen von Verbindungen mit einer chemisch recht labilen Glykosidbindung sind, zumindest beim Arbeiten im Mikrobereich, Bedenken angebracht. 
Für die von Zhivkov (5) beschriebene Methode, nach der Leber mit Perchlorsäure homogenisiert, danach ein Nucleotidrohextract durch Chromatographie über einen Ionenaustauscher hergestellt, dieser papierchromatographisch aufgetrennt und die isolierte UDP-Glucuronsäure UV-spektroskopisch bestimmt wird, ist $1 \mathrm{~g}$ Leber/Ansatz erforderlich. Die Dauer einer Bestimmung beträgt mindestens 3 Tage.

Keppler et al. (6) adsorbieren die Nucleotide an Holzkohle, trennen sie nach Auswaschen papierchromatographisch und bestimmen sie sodann enzymatisch. Auch hier werden $1 \mathrm{~g}$ Lebergewebe benötigt und die Prozedur dauert drei bis vier Tage. Zuletzt sei eine weitere Mikromethode von Wong (7) erwähnt, bei der die UDP. Glucuronsäure an $\left[{ }^{14} \mathrm{C}\right]$ Tyramin gekoppelt, das Glucuronid papierchromatographisch abgetrennt, seine Radioaktivität gemessen und die entsprechende UDP-G̈lucuronsäure-Menge an einer mit der jeweiligen Mikrosomenpräparation sich ändernden Standardkurve abgelesen wird. Zur Durchführung der Bestimmung werden immer noch $60 \mathrm{mg}$ Gewebe benötigt. Einschränkend muß gesagt werden, daß auch hier der Gewebsextrakt durch Kochen gewonnen wird. Die Dauer der Bestimmung beträgt $24 \mathrm{~h}$.

Bei der von uns entwickelten Methode zur Bestimmung der UDP-Glucuronsäure wird ein alkoholischer Leberextrakt nach Entfernen des Alkohols mit gewaschenen Meerschweinchenmikrosomen als UDP-Glucuronyltransferase-Quelle und einer bekannten Menge an $\left[{ }^{14} \mathrm{C}\right] p$ Nitrophenol inkubiert und die UDP-GlucuronsäureKonzentration nach Extraktion und dünnschichtchromatographischer Trennung des überschüssigen $\left[{ }^{14} \mathrm{C}\right] p$-Nitrophenols von seinem Glucuronid berechnet. Zur Korrektur des nicht vollständigen UDP-Glucuronsäure-Umsatzes und der während der Prozedur eventuell auftretenden Verluste wird ein zweiter Bestimmungsansatz mitgeführt, dem am Anfang außer dem Zellextrakt eine bekannte Menge an UDP-Glucuronsäure zugesetzt worden ist.

Bezüglich der biochemischen und chromatographischen Bedingungen konnten wir auf eigene gute Erfahrungen im Zusammenhang mit der Entwicklung einer Mikromethode zur Bestimmung der UDP-Glucuronyltransferase zurückgreifen (8). Mit der hier beschriebenen Methode lassen sich an einem Arbeitstag 6 und mehr Proben bewältigen.

\section{Material und Methoden}

\section{Chemikalien}

UDP-Glucuronat, di-Natriumsalz; Boehringer Mannheim. [ $\left.{ }^{14} \mathrm{C}\right] p$ Nitrophenol, spez. Aktivität $2,98 \times 10^{8} \mathrm{~Bq} / \mathrm{mmol}(8,06 \mathrm{mCi} /$ mmol), ICN Tracerlab, über Amersham-Buchler, Braunschweig. $p$-Nitrophenyl- $\beta$ - $D$-glucuronid, $p$-Nitrophenyl- $\beta$ - $D$-xylopyranosid, $p$-Nitrophenyl- $\beta-D$-galaktopyranosid, Serva Entwicklungslabor, Heidelberg.
$p$-Nitrophenyl- $\beta$ - $D$-glucosid, $p$-Nitrophenyl-N-acetyl- $\beta$ - $D$-glucosaminid, Sigma Chemie GmbH, München.

UDP-Glucose, -Xylose, -Galaktose, -N-Acetylglucosamin als NaSalze, Sigma Chemie, München.

p-Nitrophenol p.a., Eisessig p.a., 2.5-Diphenyloxazol (PPO), 2.2'-p-Phenylen-bis-(5-phenyloxazol) (POPOP), Naphthalin für die Szintillationsmessungen, Methanol p.a., Dioxan p.a., Cellulose-F-Dünnschichtplatten $10 \times 20 \mathrm{~cm}$, E. Merck, Darmstadt, Hyamine-10-X-Hydroxyde, $1 \mathrm{~mol} / \mathrm{l}$ in Methanol, Packard Instr. Frankfurt/M.

Ethanol absol. (vgl. l.c. (8)).

\section{Lösungen}

Inkubationslösung: $10 \mathrm{ml}$ Kaliumchlorid-Lösung ( $66 \mathrm{mmol} / \mathrm{l}$ ), $3 \mathrm{ml}$ Natriumphosphatpuffer ( $200 \mathrm{mmol} / \mathrm{l}, \mathrm{pH} 7,4), 1 \mathrm{ml} \mathrm{Mag}$ nesiumchlorid-Lösung ( $300 \mathrm{mmol} / \mathrm{l})$ und $10 \mathrm{ml}$ isotone alkalische Kaliumchlorid-Lösung (154 mmol/1 Kaliumchlorid und 0,32 mmol/1 Kaliumhydrogencarbonat).

UDP-Glucuronat-Lösung: $3,75 \mathrm{mg} / 1$ 86\% di-Natrium-UDPglucuronat in bidest. Wasser $=5,16 \mu \mathrm{mol} / 1$.

p-Nitrophenol/p-Nitrophenylglucuronid-Gemisch: $1 \mathrm{ml} p$-Nitrophenol-Lösung ( $1 \mathrm{mmol} / 1$ in bidest. Wasser) $0,1 \mathrm{ml} p$-Nitrophenyl- $\beta$ - $D$-glucuronid-Lösung $(1 \mathrm{mmol} / \mathrm{l}), 2 \mathrm{ml}$ Salzsäure ( 25 $\mathrm{mmol} / \mathrm{l}$ ) und $6 \mathrm{ml}$ bidest. Wasser.

Diatol: 3,25 g PPO, $65 \mathrm{mg}$ POPOP, $65 \mathrm{~g}$ Naphthalin, $150 \mathrm{ml}$ Methanol, $250 \mathrm{ml}$ Toluol, $250 \mathrm{ml}$ Dioxan.

Geräte

Potter-Elvehjem-Glashomogenisator, 50, 25, 15 und $2 \mathrm{ml}$.

Motor mit konstanter und reproduzierbar einstellbarer Umdrehungszahl, Cole-Parmer Instr. Comp., Chicago, Ill. 60648.

Labor-Kühlzentrifuge mit Ausschwingrotor, $R_{a v}=17,5 \mathrm{~cm}$ und Kühlzentrifuge, Zeta 20 mit Festwinkelrotor, $R_{a v}=11,5 \mathrm{~cm}$, Heraeus-Christ, Osterode.

Ultrazentrifuge, Spinco L2 65B mit Festwinkelrotor $50 \mathrm{Ti}$, $R_{\mathrm{av}}=5,9 \mathrm{~cm}, 40000 \mathrm{U} / \mathrm{min}=105000 \mathrm{~g}$, Beckman Instr., Fullerton Calif., U.S.A. Rotationsverdampfer mit "Spinne“, sechs- und zwölfarmig, NS 14,5, Büchi, Flavil, Schweiz.

Präzisionsspritzen, $50 \mu 1$, Hamilton, Whittier, Calif., U.S.A. Flüssig-Szintillationsspektrometer, TriCarb, Mod. 3375, Packard Instr., Frankfurt/M.

Schwingmühle mit Edelstahl-Mahlkapsel und -Kugel, Bodenseewerke Perkin-Elmer, Überlingen.

\section{Durchführung der Versuche}

Mikrosomenpräparation

Das Mikrosomenpräparat wurde aus Meerschweinchenleber nach Schneider \& Hogeboom (9) gewonnen. Nach zweimaligem Waschen mit frischer, eiskalter Inkubationslösung verteilt man $1 \mathrm{~g}$ Mikrosomen durch Homogenisieren $\left(1 \mathrm{~min}, 300 \mathrm{U} / \mathrm{min}, 1^{\circ} \mathrm{C}\right.$ ) in $40 \mathrm{ml}$ Inkubationslösung und friert diese Suspension in kleinen Portionen bei $-20^{\circ} \mathrm{C}$ ein.

\section{Gewebeextrakt}

Einem in Ätheranaesthesie dekapitierten und entbluteten Versuchstier entnimmt man möglichst schnell ein Stïck Leber und friert es zwischen żwei auf Temperăturen des flüssigen Stickstoffes gebrachte Glasplatten ein (zwei mit den Böden aufeinanderstehende Petri- oder Abdampfschalen, deren obere mit flüssigem Stickstoff gefullt ist). Biopsiezylinder werden in eiskalte physiologische NaCl-Lösung gespritzt, mit einer Präpariernadel aufgelesen, auf einem Filterpapierstreifen abgetropft und anschließend sofort wie oben beschrieben auf $-196^{\circ} \mathrm{C}$ gebracht. Man wiegt die eingefrorenen Leberproben auf einem Stück tiefgefrorenen Parafilims schnell aus und bringt sie sofort wieder zwischen, die gekühlten Glasplatten oder direkt in einen Glashomogenisator mit $2 \mathrm{ml}$ auf $-40^{\circ} \mathrm{C}$ vorgekühltem absolutem Ethanol. Es wird nun ohne weitere Kühlung bei $500 \mathrm{U} / \mathrm{min}$ 5 min homogenisiert, wobei man zunächst das harte Gewebestück zerbricht und die Bruchstïcke möglichst vor dem Anstei- 
gen der Temperatur auf $0^{\circ} \mathrm{C}$ zermahlt. Man überfuhrt die Suspension in ein 10-ml-Zentrifugenglas mit NS 14,5 und spitzem Boden, spült Homogenisator und Stempel dreimal sorgfältig mit Ethanol ab und dampft im Wasserstrahlvakuum bei $30^{\circ} \mathrm{C} \mathrm{am}$ Rotationsverdampfer zur Trockene ein. Das trockene, denaturierte Leberhomogenat wird mit $40 \mu \mathrm{l}$ bidest. Wasser je mg Leberfrischgewicht versetzt, gut durchmischt und anschließend in der Laborzentrifuge zentrifugiert $\left(5 \mathrm{~min}, 3000 \mathrm{U} / \mathrm{min}, 1^{\circ} \mathrm{C}\right)$. Der Überstand (Gewebeextrakt) enthält in $100 \mu \mathrm{l}$ die UDPGlucuronsäure aus $2,5 \mathrm{mg}$ Leber.

\section{Inkubation}

Man pipettiert in 4 mit Eiswasser gekühlte Zentrifugengläser jeweils $100 \mu 1$ Mikrosomensuspension, $100 \mu$ l Gewebeex trakt und in zwei der Röhrchen, die man als ,Kalibrierbestimmung “ kennzeichnet, zusätzlich je $100 \mu$ l UDP.Glucuronsäure-Lösung (516 pmol). Anschließend werden mit einer Mikroliterspritze zu jeder Probe präzise $30 \mu 1\left[^{14} \mathrm{C}\right] p$-Nitrophenol-Lösung $(14,88$ nmol) hinzugegeben, die Röhrchen mit Parafilm verschlossen und in einem Schüttelwasserbad bei einer Neigung von ca. $30^{\circ} \mathrm{C}$ $60 \mathrm{~min}$ bei $37^{\circ} \mathrm{C}$ inkubiert.

\section{Aufarbeitung der Proben}

Man beendet die Inkubation durch Kühlen der Gläschen mit Eiswasser und sofortige Zugabe von je $2 \mathrm{ml}$ absol. Alkohol. Die Proben werden mit je $1 \mathrm{ml} p$-Nitrophenol/p-Nitrophenylglucuronid-Lösung versetzt, auf eine Spinne gesteckt, $10 \mathrm{~min}$ mit Eiswasser vorgekühlt und an den Rotationsverdampfer gehängt. Um ein Verspritzen der Proben zu vermeiden, läßt man die Spinne so lange in der Luft rotieren, bis sich das volle Wasserstrahlvakuum eingestellt hat (etwa $5 \mathrm{~min}$ ), taucht dann in ein $30^{\circ} \mathrm{C}$ warmes Wasserbad und dampft zur Trockene ein. Der Rückstand wird in $0,5 \mathrm{ml}$ absol. Ethanol aufgenommen, wobei die gesamte Innenwand des Röhrchens zu benetzen ist. Nach kurzem Zentrifugieren $\left(5 \mathrm{~min}, 3000 \mathrm{U} / \mathrm{min}, 1^{\circ} \mathrm{C}\right.$ ) hebert man, ohne den Bodensatz zu berühren (!), je $0,4 \mathrm{ml}$ vom Uberstand in ein zweites Röhrchen, dampft wie oben beschrieben zur Trockene ein, nimmt den Rückstand in je $50 \mu \mathrm{l}$ absol. Alkohol auf, wobei die untere Hälfte der Röhrchen benetzt werden sollte und zentrifugiert kurz wie oben. Vom Uberstand werden je $20 \mu \mathrm{l}$, der Bodensatz soll auch hier nicht mit der Pipette berührt werden, in etwa 4 Portionen auf Cellulose-F-Dünnschichtplatten $2,5 \mathrm{~cm}$ vom linken und unteren Rand aufgetragen und gründlich getrocknet. Man chromatographiert anschließend etwa $3 \mathrm{~h}$ mit Wasser/ Eisessig (Volumina, $100 \mathrm{ml}+1 \mathrm{ml}$ ).

\section{Auswertung}

Um die im UV-Licht sichtbaren $p$-Nitrophenol-( $R_{F}$ ca. 0,6) und p-Nitrophenylglucuronid-Flecke $\left(R_{F} c .0,9\right)$ zeichnet man mit Bleistift Rechtecke von $3 \times 2,5$ bzw. $3 \times 3 \mathrm{~cm}$ (hier, ohne die Lösungsmittelfront mit einżubeziehen), schabt die markierten Areale mit einem Spatel ab, überführt die Cellulose mit Hilfe von Glanzpapier in PVC-Szintillationsröhrchen und versetzt mit je $1 \mathrm{ml}$ Hyamine-10-X-Hydroxyd-Lösung (schütteln), 0,1 ml Eisessig (schütteln) und $10 \mathrm{ml}$ Diatol (schütteln). Nach Abklingen evtl. vorhandener Eigenluminiszenz wird im Flüssigkeitsszintillationsspektrometer gezählt. Die UDP-GlucuronsäureMenge errechnet sich nạch folgender Gleichung:

\section{UDPGICUA (vorh.) =}

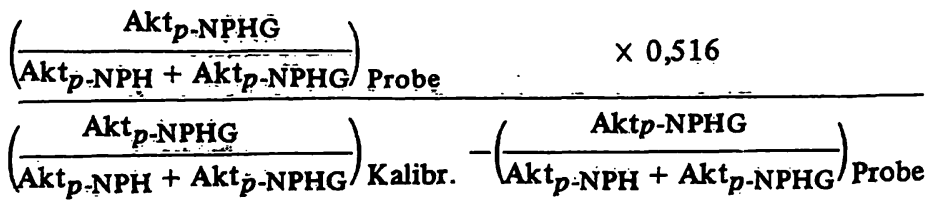

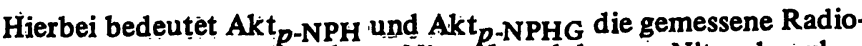
aktivität der entsprechenden $p$-Nitrophenol-bzw. $p$-Nitrophenylglucuronid-Flecken. 0,516 ist die zur Kalibrierprobe zugesetżte Menge an UDP-Glucuronsäure in nmol/2,5 mg Gewebe. Die in der Probe vorhandene und zu messende. UDP-Glucuronsäure-Menge UDPGICUA vorh. hat die Dimensiọn numol/2,5 mg. Gewebe.

\section{Ergebnisse und Diskussion}

Nachdem die Entwicklung einer Mikromethode zur Bestimmung der UDP-Glucuronyltransferase-Aktivität mit $p$ Nitrophenol als Substrat (8) hinsichtlich der Reproduzierbarkeit zu guten Ergebnissen geführt hatte, war es naheliegend, diese Erfahrungen auch für eine Mikromethode zur Bestimmung der UDP-Glucuronsäure zu nutzen. Nach ersten Vorversuchen zeigte sich, daß ein vollständiger Umsatz der UDP-Glucuronsäure zum $p$-Nitrophenylglucuronid nicht zu erreichen war, da offenbar ein nicht unwesentlicher Anteil im Inkubationsansatz entweder anderweitig verbraucht oder nicht umgesetzt wurde. Für Nebenreaktionen könnten die UDP-Glucuronat-Pyrophosphatase sowie die $\beta$-Glucuronidase verantwortlich gemacht werden. Wie Pogell et al. (10) gezeigt haben, ist die UDP-Glucuronat-Pyrophosphatase, deren $\mathrm{pH}$-Optimum bei pH 9 liegt, in Meerschweinchenmikrosomen kaum nachweisbar. Die Aktivität der $\beta$-Glucuronidase, mit einem pH-Optimum von $\mathrm{pH} 5,0$ ist bei einem $\mathrm{pH}$ von 7,4 zu vernachlässigen (11). Um die während der Inkubation wie auch immer eintretenden UDP-Glucuronsäure-Verluste zu berücksichtigen, mußte ein Kalibrierverfahren eingefuhrt werden. Wir ließen daher parallel zu den Bestimmungsansätzen Kalibrieransätze laufen, denen bekannte Mengen an UDP-Glucuronsäure zugemischt wurden, die sonst aber mit den Bestimmungsansätzen tdentisch waren. Im gemessenen Bereich zwischen 0 und 1,45 nmol UDP-Glucuronsäure/Ansatz erhielten wir eine Gerade, die durch den Nullpunkt geht (Abb. 1) und der Beziehung $\mathrm{y}=1,9 \mathrm{x}$ gehorcht. Der Bestimmtheitsgrad $r^{2}$ (Maß für die Güte der Anpassung) beträgt 0,9998. Eine spätere Wiederholung dieses wichtigen Versuches

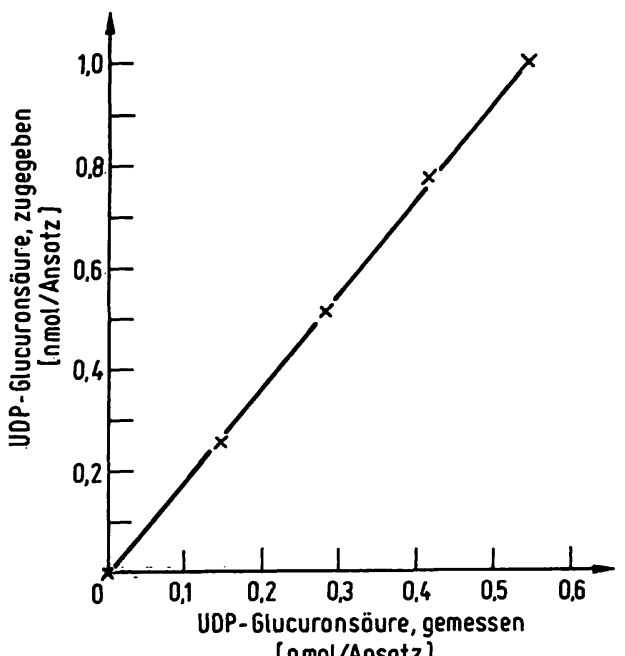

Abb. 1. Abhängigkeit der gefundenen von der eingesetzten UDPGlucuronsäure-Menge.

Inkubationsansatz: Es werden 0-1,03 nmol UDP-Glucuronsäure mit je $100 \mu$ l Gewebeextrakt, $30 \mu l\left[{ }^{14} \mathrm{C}\right]$ $p$-Nitrophenol-Lösung und $100 \mu 1$ Mikrosomensuspension nebst einem Leerwert (ohne UDP-Glucuronsäure-Zugabe und ohne Gewebeextrakt) $1 \mathrm{~h}$ bei $37^{\circ} \mathrm{C}$ inkubiert.

Es resultiert eine Gerade, die durch den Nullpunkt geht $(y=1,9 x)$. Zur Korrektur der Meßwerte ist daher jeweils ein Kalibrierpunkt ausreichend. 
mit einer anderen Mikrosomenpräparation und von einer anderen Person durchgeführt, ergab ebenfalls eine Gerade durch den Nullpunkt ( $\left.y=1,6 x ; r^{2}=0,997\right)$. Aus diesen Versuchen ergibt sich, daß das Erstellen von Standardkurven nicht notwendig ist. Es reicht ein mit jeder Bestimmung mitzuführender Kalibrierpunkt.

Um auszuschließen, daß neben UDP-Glucuronsäure im Gewebeextrakt möglicherweise ebenfalls vorhandene UDP-Glucose, -Xylose, -Galaktose und -N-Acetylglucosamin fälschlicherweise als UDP-Glucuronsäure mitbestimmt werden, wurden diese Nucleotide mit $\left[{ }^{14} \mathrm{C} p\right.$ Nitrophenol und Mikrosomen wie üblich inkubiert. Parallel dazu liefen zur Kontrolle Ansätze mit UDPGlucuronsäure. Wie die autoradiographische Auswertung und Messungen der Dünnschichtplatten mit einem Dünnschichtscanner zeigten, wurde zwar immer $p$ Nitrophenylglucuronid, nie aber Konjugat der Zucker mit $p$-Nitrophenol gefunden. Zu gleichen Ergebnissen kommt auch Wong (7) mit $\left[{ }^{14} \mathrm{C}\right]$ Tyramin als Substrat.

Um zu prüfen, welche Art der Gewinnung des Gewebeextraktes die geeignetere ist, haben wir aus zwei benachbarten Leberstückchen der Maus einen Extrakt wie üblich und einen durch Kochen ( $3 \mathrm{~min}$ ) in bidest. Wasser gewonnen und die UDP-Glucuronsäure bestimmt. Während nach Denaturierung mit Alkohol in der Kälte $172 \mu \mathrm{mol}$ gemessen wurden, enthielt die gekochte Probe nur noch $128 \mu \mathrm{mol} / \mathrm{kg}$ UDP-Glucuronsäure, also ca. $25 \%$ weniger! Wir halten daher, zumindest bei Mikrobestimmungen, das Kochen von Gewebsproben zur Extraktion von UDP-Glucuronsäure für unzulässig.

Um zu klären, ob nicht schon während des Ausblutens des Tieres und des Einfrierens der Leberprobe ein Teil der unter physiologischen Bedingungen vorhandenen UDPGlucuronsäure verloren geht, haben wir je zwei ätherbetäubten Mäusen nach Laparotomie je ein Stück Leber in situ durch Quetschen zwischen zwei $-196^{\circ} \mathrm{C}$ kalte Platten eingefroren und entnommen und nach Dekapitieren und Entbluten der Tiere je eine Probe wie üblich gewonnen. Tabelle 1 (Maus A und B) zeigt, daß ein Einfrieren der Proben in situ nicht notwendig ist.

Hingegen nimmt der UDP-Glucuronsäure-Gehalt von Leberstïckchen, die verschieden lange in physiologischer $\mathrm{NaCl}$-Lösung bei Raumtemperatur aufbewahrt werden, sehr schnell ab (Abb. 2). Nach 3 min ist nur noch 63\% der anfänglichen Menge vorhanden. Proben sollten also unmittelbar nach der Entnahme tiefgefroren werden.

Wie aus Abbildung 3 ersichtlich, bleibt die UDP-Glucuronyltransferase-Aktivität von gewaschenen Meerschweinchenmikrosomen beim Aufbewahren bei $-20^{\circ} \mathrm{C}$ über 12 Wochen nahezu konstant, um dann langsam abzufallen.

Tabelle 1 zeigt die gute Ubereinstimmung der Werte bei Mehrfachbestimmungen. Der aus 20 Doppelbestim-
Tab. 1. Gemessene UDP-Glucuronsäure-Konzentrationen in Mäuseleber (bei Einfrieren der Proben nach Entnahme bzw. in situi) und in der Leber von zwei Patienten. Es handelt sich jeweils um den Mittelwert von Doppelbestimmungen.

\begin{tabular}{|c|c|c|c|}
\hline \multirow{2}{*}{\multicolumn{2}{|c|}{$\begin{array}{l}\text { Untersuchungs- } \\
\text { material }\end{array}$}} & \multicolumn{2}{|c|}{$\begin{array}{l}\text { UDP:Glucuronsäure-Gehalt der Leber } \\
{[\mu \mathrm{mol} / \mathrm{kg}]}\end{array}$} \\
\hline & & $\begin{array}{l}\text { Nach Entnahme } \\
\text { eingefroren }\end{array}$ & $\begin{array}{l}\text { In situ } \\
\text { éingefrororen }\end{array}$ \\
\hline Maus & $\begin{array}{l}\text { A } \\
\text { B } \\
\text { C } \\
\text { D } \\
\text { E } \\
\text { E } \\
\text { F } \\
\text { F }\end{array}$ & $\begin{array}{l}170 \pm 2 \\
168 \pm 3 \\
152 \pm 4 \\
201 \pm 2 \\
164 \pm 1 \\
168 \pm 2 \\
170 \pm 1 \\
172 \pm 3\end{array}$ & $\begin{array}{l}168 \pm 3 \\
169 \pm 2\end{array}$ \\
\hline \multicolumn{2}{|c|}{$\begin{array}{l}\text { Patient M.D., } ९ \text {, } \\
6 \text { Wochen alt } \\
\text { Patient O.E., o, } \\
57 \text { Jahre alt }\end{array}$} & $\begin{array}{l}142 \pm 3 \\
192 \pm 4\end{array}$ & $\cdot$ \\
\hline
\end{tabular}

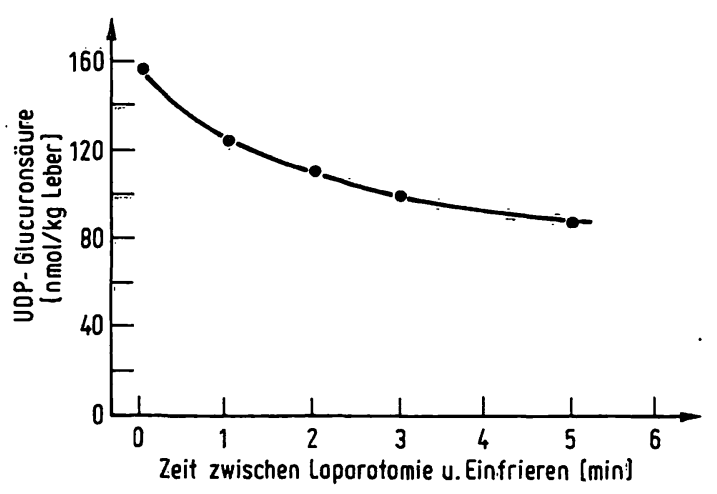

Abb. 2. Effekt dẹr Lagerung von Leberproben in physiologischer $\mathrm{NaCl}-\mathrm{Lösung}$ bei Raumtemperatur auf den UDP-Glucuronsäure-Gehalt. Der Versuch zeigt, daß die Zeit zwischen Laparotomie bzw. Punktion und Ėinfrieren der Probe kurz gehalten werden muß.

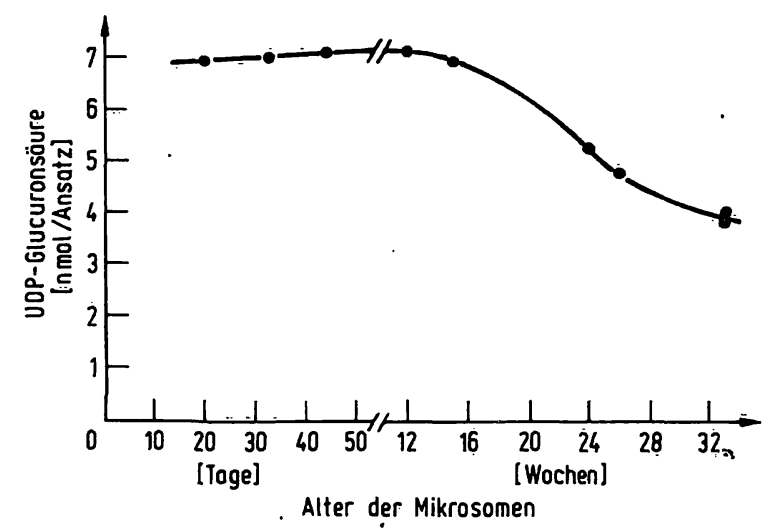

Abb. 3. Einfluß der Lagerung der Meerschweinchenmikrosomen bei $-20^{\circ} \mathrm{C}$ auf den Umsatz von UDP-Glucuronsäure mit p-Nitrophenol. Die Mikrosomen können ohne Aktivitätsverlust mindestens 12 Wochen verwendet werden.

mungen nach logarithmischer Transformation ermittelte Variationskoeffizient beträgt $\pm 2,3 \%$. Die Wiederfin: dung liegt zwischen 96 und 103\%.(Tab. 2). 
Tab. 2. Versuche zur Richtigkeit der Analysen. Die Zahlen belegen die Richtigkeit der Ergebnisse über einen weiten Konzentrationsbereich.

\begin{tabular}{lll}
\hline $\begin{array}{l}\text { UDP-Glucuronsäure [nmol/Probe] } \\
\text { eingesetzt }\end{array}$ & Wiederfindung \\
\hline 0,258 & 0,265 & 1,03 \\
0,774 & 0,797 & 1,03 \\
1,032 & 1,058 & 1,03 \\
1,546 & 1,582 & 1,02 \\
2,064 & 1,990 & 0,96 \\
\hline
\end{tabular}

In der Mäuseleber wurden UDP-Glucuronsäure-Werte zwischen 164 und $201 \mu \mathrm{mol} / \mathrm{kg}$ gefunden.

Der UDP-Glucuronsäure-Gehalt in menschlichem Lebergewebe konnte in zwei Fällen bestimmt werden (Tab. 1). Beide Gewebsproben wurden während einer Laparotomie entnommen. M.D. hatte eine Hyperbilirubinämie, die auf einen Morbus Crigler-Najjar beruhte, d.h. auf einen angeborenen Defekt der UDP-Glucuronyltransferase.

\section{Literatur}

1. Hess, R. \& Pearse, A. G. (1961), Experientia 17, 317-318.

2. Felsher, B. F., Maidman, J. E., Carpio, N. M., Van Couvering, K. \& Woolley, M. M. (1978), Pediatr. Res. 12, 838-840.

3. Schröter, W. \& Eggerling, U. (1965), Klin. Wochenschr. 43, 829-834.

4. Wong, K. P. \& Sourkes, T. L. (1967), Anal. Biochem. 21, $444-453$.

5. Zhivkov, V. (1970), Biochem. J. 120, 505-508.

6. Keppler, D.O.R., Rudigier, J. F. M., Bischoff, E. \& Decker, K. F. A. (1970), Eur. J. Biochem. 17, 246-253.
7. Wong, K. P. (1977), Anal. Biochem. 82, 559-563.

8. Lehnert, W., Limberg, J. \& Künzer, W. (1974), Z. Klin. Chem. Klin. Biochem. 12, 23-27.

9. Schneider, W. C. \& Hogeboom, G. H. (1960), J. Biol. Chem. $183,123-128$.

10. Pogell, B. M. \& Leloir, L. F. (1961), J. Biol. Chem. 236, 293-298.

11. Dutton, G. J. (1966), Glucuronic Acid, Free and Combined. Academic Press New York and London S. 249.

Dr. W. Lehnert Univ.-Kinderklinik Mathildenstr. 1 D-7800 Freiburg/Br. 

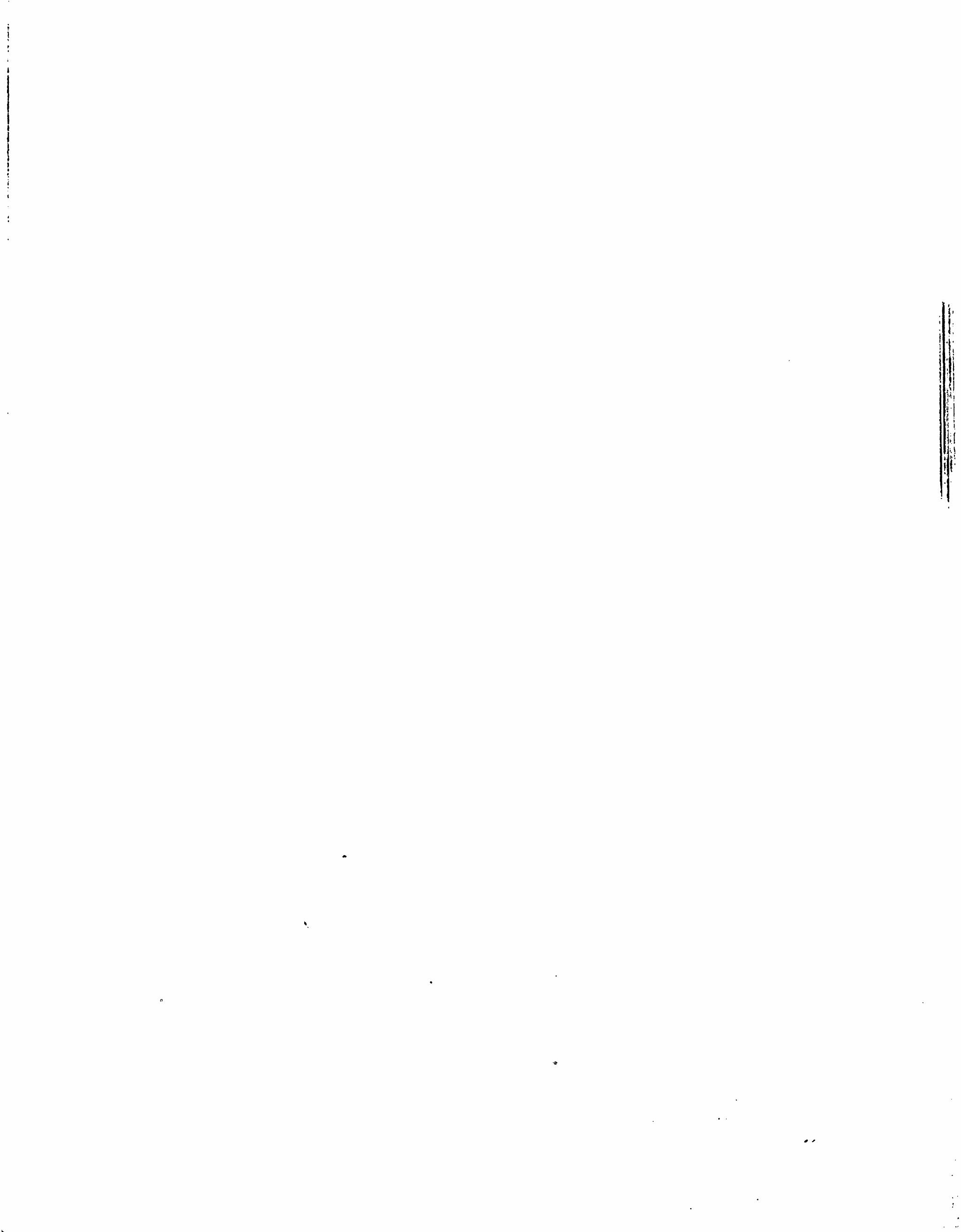